\title{
AS ÁREAS ÚMIDAS DO OESTE DE MINAS GERAIS: O DESENVOLVIMENTO AGRÍCOLA E AS ALTERAÇÕES DE CONCENTRAÇÃO DE MACRO E MICRONUTRIENTES NO TOPO DA CHAPADA DO MUNICÍPIO DE UBERLÂNDIA/MG
}

\author{
Daniele Araújo Ferreira $^{(\mathrm{a})}$, Willian Ferreira Alves ${ }^{(\mathrm{b})}$, \\ (a, b) Instituto de Geografia, Universidade Federal de Uberlândia, danielearaujo0379@ gmail.com
}

\author{
Eixo: SOLOS E PAISAGEM
}

\begin{abstract}
Resumo
Expansão e intensificação são dois caminhos para aumentar a produtividade agrícola, no entanto ambos causam impactos ambientais. No oeste de Minas Gerais, a expansão e a intensificação da agricultura iniciada na década de 1970 ocuparam, inicialmente, as áreas com Latossolos, mas a necessidade de mais terras para cultivo excedeu seus limites e avançou ou incorporou as áreas úmidas de campos de murundus. Esses campos de murundus correspondem a depressões topográficas localizadas na superfície da chapada sedimentar, pois são ecossistemas que permanecem temporária ou permanentemente alagados. Possuem solos com horizonte superficial rico em matéria orgânica sobreposto a horizonte mineral cinza-claro ou mosqueado, refletindo as condições de redução do óxido de ferro em ambientes alagados. Diante do exposto, o objetivo deste trabalho será de avaliar os impactos gerados pela agricultura em áreas de murundus mediante comparação dos teores de macronutrientes $(\mathrm{Ca}$ e $\mathrm{Mg})$ presentes em um murundu drenado e usado para cultivo e outro não impactado e considerado como solo de referência. Espera-se com esta pesquisa contribuir de forma direta na prevenção de impactos gerados através do uso intenso de agrotóxicos e fertilizantes em áreas de solos hidromórficos.
\end{abstract}

Palavras chave: Cerrado. Campus de Murundus. Solos hidromórficos. Fertilidade do solo. Uberlândia/MG

\section{INTRODUÇÃO}

As regiões tropicais do planeta tem sido o local onde a expansão agrícola tem aumentado sem encontrar qualquer tipo de limitação ou dificuldade. Estima-se que $80 \%$ das novas áreas de cultivo estejam substituindo vegetações originais destas regiões (GIBS et al., 2010).

A intensidade e rapidez com que vem acontecendo tal expansão é algo preocupante, uma vez que os biomas tropicais são grandes reservatórios de biodiversidade, além de possuírem grande estoque de carbono no solo.

Estudos científicos apontam que nas últimas cinco décadas dobraram-se as áreas agrícolas irrigadas no planeta (FAO, 2011; ROSEGRANT, 2002; GLEICK, 2003), ao passo que o uso global de fertilizantes aumentou em 500\%, e, se considera apenas o nitrogênio, o aumento foi de mais de 800\% (FAO, 2011; MATSON et al., 1987; TILMAN et al., 2001). A intensificação e expansão dessas áreas agricultáveis também tem causado degradação e contaminação das águas, aumento do uso de energia e poluição generalizada (MATSON et al., 1987; VOROSMARTY et al., 2000; DIAZ; ROSEMBENRG, 2008). 
Dentre os impactos ambientais causados em áreas agrícolas, pode-se incluir aqueles que são causados pela expansão (onde lavouras e pastagens se estendem para novas áreas, substituindo os ecossistemas naturais) e os que são causados pela intensificação (terras já utilizadas são gerenciadas para serem mais produtivas, muitas vezes através do uso de irrigação, fertilizantes, biocidas e mecanização). Quando se trata da expansão agrícola, esta tem ocasionado relevantes impactos sobre os habitats, biodiversidade, armazenamento de carbono e as condições do solo (Foley et al., 2011).

De acordo com Gleick (2009) e Postel (1999), cerca de 70\% da água doce mundial é destinada a irrigação. Além disso, o excessivo uso de fertilizantes, a aplicação de esterco e a utilização de leguminosas têm causado modificações nos ciclos globais do nitrogênio e do fósforo (VITOUSEK et al., 1997; BENNETT et al., 2001), acarretando impactos na qualidade da água, nos ecossistemas aquáticos e na pesca marinha (DIAZ, 2008; CANFIELD, 2010).

No Brasil, as atividades relacionadas ao agronegócio têm sido responsáveis pela geração de grande riqueza econômica e ao mesmo tempo tem sido responsáveis por grandes alterações de cunho ambiental nos biomas brasileiros, e no Cerrado não é diferente. Neste bioma também conhecido como "Savanas Brasileiras", estão presentes as áreas úmidas (AU's), ecossistemas presentes nas paisagens tropicais que são particularmente sensíveis aos impactos agrícolas. As AU's são áreas "onde a água é o principal fator controlador do ambiente, juntamente com a fauna e a flora associadas" (NIERING, 1985).

Estas áreas são prioritárias para a conservação, por prestarem vários serviços ambientais, como o fornecimento e o armazenamento de água, a regularização do fluxo e da vazão na bacia hidrográfica, na retenção de sedimentos e contaminantes provenientes da agricultura, fornecimento da turfa e armazenamento de carbono. Também são áreas que abrigam fauna e flora, inclusive com espécies endêmicas (MATIZA, 1994; MITSCH e GOSSELINK, 2007).

No Planalto Central Brasileiro localiza-se a Chapada Sedimentar de Uberlândia/Uberaba-MG, originalmente recoberta pelo Cerrado strictu sensu, onde estão presentes importantes AU's classificadas regionalmente como veredas e/ou campus de murundus. Estas AU's possuem expressivo número de nascentes que abastecem as cidades desta chapada inclusive o maior município que é Uberlândia. Dentre os principais rios encontram-se o Ribeirão Bom Jardim e o Rio Uberabinha, área de estudo deste trabalho. Esta microrregião foi rapidamente convertida em área agrícola, tornando-se, ao mesmo tempo, um local de expressiva produção de commodities e grande passivo ambiental, principalmente no que diz respeito ao desmatamento e a conversão e degradação das áreas úmidas

As atividades agrícolas como é sabido geram impactos ambientais geralmente negativos e por vezes irreversíveis ao ambiente, como por exemplo, o ressecamento de nascentes, drenagem de terrenos, erosão do solo, cavas provocadas pela mineração e o garimpo (SCHENEIDER, 1996; BRITO, 2001; SOARES, 2008; COSTA, 2009; QUEIROZ, 2012; BORGES, 2012; FERREIRA, 2015a) 
Quando se considera o compartimento de chapadas do Oeste Mineiro, a análise do uso do solo indica que tem ocorrido grande ampliação das atividades agrícolas das áreas de Latossolo em direção às áreas hidromórficas (veredas e murundus). Com o avanço das lavouras, foi constatado o aumento das concentrações dos elementos químicos nocivos no solo, presentes em fertilizantes e agrotóxicos, relatados em trabalhos como de FERREIRA (2015b).

Os campos de murundus típicos do alto da chapada mencionada, caracterizam-se por serem locais de alta permeabilidade e baixa drenagem, a intensa produção de commodities nesta área tem ocasionado uso de elevadas concentrações tanto de fertilizantes quanto de agrotóxicos, que estão sendo depositados e consequentemente acumulados nos solos hidromórficos desta microrregião do Oeste mineiro.

Trabalhos anteriores forneceram análises qualitativas e quantitativas relacionadas à incorporação das áreas úmidas da superfície da chapada Uberlândia/Uberaba-MG pela agricultura, onde pode ser verificado em Schneider (1996), Ramos et al., (2006), Soares (2008), Resende (2011), Borges (2012), Queiroz (2012), Oliveira (2013), Machado (2014).

As AU's se caracterizam como ambientes óxido-redutores e com elevado teor de matéria orgânica. Os Gleissolos presentes nos campos de murundus podem ser áreas de interceptação e de acumulação de elementos químicos usados na agricultura, sendo grandes responsáveis por desequilíbrios nos ciclos biogeoquímicos e impactos negativos causados nos ambientes terrestres e aquáticos.

Análises químicas totais dos solos fornecem informações valiosas sobre a elevação da concentração de elementos nutritivos usados na agricultura e servem como parâmetros para a determinação da extensão dos impactos da atividade econômica no ambiente. Esses dados podem orientar os estudos da potencialidade de ocorrência de problemas nutricionais (em plantas e animais), de saúde e ambientais (GUPTA, 1991) e justificam este trabalho.

\section{OBJETIVOS}

\subsection{Objetivo Geral}

O objetivo geral deste trabalho foi de avaliar as concentrações e as propriedades morfológicas e químicas do solo hidromórficos em áreas de murundus de topo de chapada, através das concentrações dos macronutrientes cálcio $(\mathrm{Ca})$ e magnésio $(\mathrm{Mg})$ na microbacia do Córrego do Caroço afluente do Rio Uberabinha.

\subsection{Objetivos Específicos}

Analisar as propriedades morfológicas e químicas de perfis de solo hidromórfico de murundus visando comparar as mudanças ocorridas com o uso de insumos agrícolas; 
> Determinar a concentração de cálcio e magnésio macronutrientes essenciais ao solo.

\section{MÉTODOS E TÉCNICAS DE PESQUISA}

A área de estudo situa-se nos campos de murundus que compõem a nascente do Córrego do Caroço, um dos afluentes do Rio Uberabinha. Localiza-se entre as coordenadas $19^{\circ} 20^{\prime} 15^{\prime \prime}$ a $19^{\circ} 20^{\prime} 69^{\prime \prime}$ de latitude sul e $47^{\circ} 55^{\prime} 46^{\prime \prime}$ a 47055'77' de longitude oeste, a uma altitude de 974 metros.

A bacia hidrográfica que drena o topo da chapada comporta dois cursos d'água principais, o Rio Uberabinha e o Ribeirão Beija-Flor. Suas nascentes estão situadas ao Norte do Município de Uberaba e seguem em direção Sul do Município de Uberlândia, passando pelo perímetro urbano, seguindo em direção à foz no Rio Araguari, dividindo os Municípios de Uberlândia e Tupaciguara. O córrego Caroço é afluente do Uberabinha em seu alto curso.

Onde, para avaliar os impactos relacionados a atividade agrícola em solos com propriedades hidromórficas em campos de murundus foram coletadas amostras em três profundidades distintas. O alto curso da bacia do Rio Uberabinha no Triângulo Mineiro visualizado na Figura 1, foi escolhido por se caracterizar como uma área representativa de ampla extensão dos campos de murundus do cerrado do Triângulo Mineiro, ao mesmo tempo em que é ocupada por intensa atividade agropecuária.

A área encontra-se há aproximadamente $45 \mathrm{~km}$ de Uberlândia e $40 \mathrm{~km}$ de Uberaba, sendo o acesso preferencial por ambas as cidades pela BR-050. Todas as análises químicas de solo foram realizadas no Laboratório de Análise Química de Solo, Tecido Vegetal e Fertilizante da Universidade Federal de Viçosa/MG Os resultados das análises ainda serão submetidos a uma avaliação estatística a $5 \%$ de probabilidade pelo teste de Friedman e Mann-Whitney. 


$\begin{gathered}\text { XVII Simpósio Brasileiro } \\ \text { de Geografia Fisica Aplicada }\end{gathered}$
$\begin{aligned} & \text { I Congresso Nacional } \\ & \text { de Geografia Física }\end{aligned}$

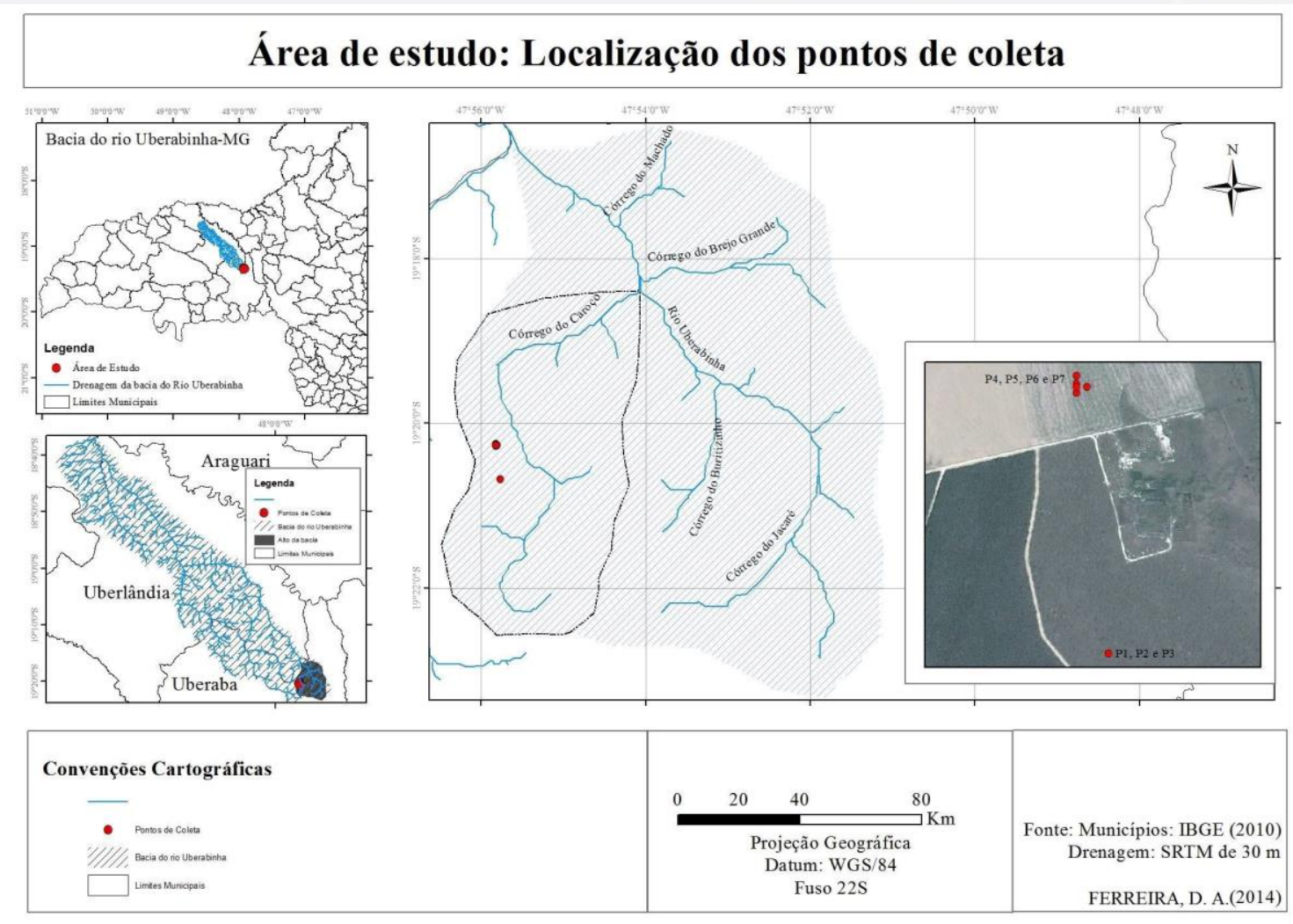

Figura 1 Localização da Área de Estudo e Pontos de Coleta Autora: Ferreira, 2014

\subsection{Coleta de Amostras}

Para avaliar os impactos relacionados com a atividade agrícola em solos com propriedades hidromórficos em campos de murundus, foram coletadas amostras de solo e comparados os resultados entre uma área de murundu drenado (MD), usado para cultivo de grãos (arroz, soja, milho e feijão); e uma área de murundu preservado considerada como solo de referência (MR) como pode ser visualizado nas Figuras $2 \mathrm{a}$ e $2 \mathrm{~b}$.
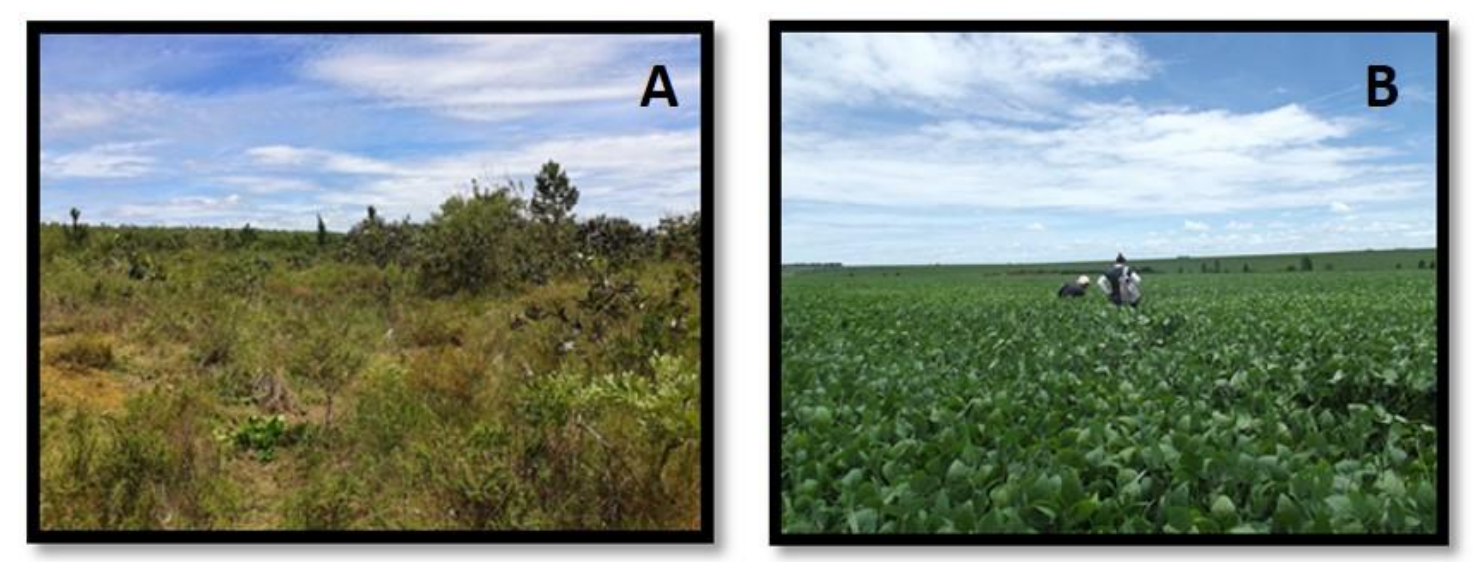

Figura 2 - A - Campus de Murundus Referência (MR); B - Área Agrícola de Murundus Drenados (MD) Autora: Ferreira, 2014 


\section{OS DESAFIOS DA GEOGRAFIA FÍSICA NA FRONTEIRA DO CONHECIMENTO \\ Instituto de Geociências - Unicamp \\ Campinas - SP \\ 28 de Junho à 02 de Julho de 2017}

As amostras foram coletadas em pontos distintos $\left(\mathrm{P}_{1}, \mathrm{P}_{2}, \mathrm{P}_{3}, \mathrm{P}_{4}, \mathrm{P}_{5}, \mathrm{P}_{6}\right.$ e $\left.\mathrm{P}_{7}\right)$ e em três profundidades (0$20 \mathrm{~cm}, 20-40 \mathrm{~cm}$ e $40-60 \mathrm{~cm}$ ). Os pontos $\mathrm{P}_{1}, \mathrm{P}_{2}$ e $\mathrm{P}_{3}$ correspondem ao murundu preservado (MR), que foi considerado como de referência para a qualidade dos solos. Os pontos $\mathrm{P}_{4}, \mathrm{P}_{5}, \mathrm{P}_{6}$ e $\mathrm{P}_{7}$ são referentes ao murundu drenado (MD), que era ocupado no momento da coleta por cultura de soja. Pode ser visualizada na Figura 3 a área no entorno dos pontos de coleta onde se detectou que grande parte da vegetação original foi retirada e substituída pelas culturas de grãos principalmente.

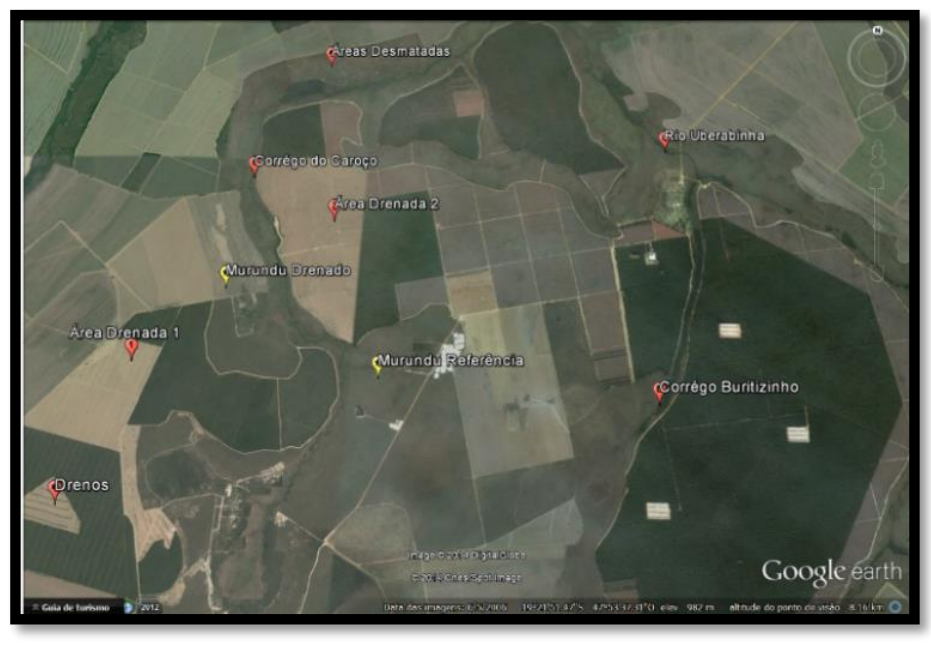

Figura 3 Área no Entorno dos Pontos de Coleta

Fonte: Google Earth, 2014

Organização: Ferreira, 2014

\subsection{Análise Química}

\subsubsection{Análise do Cálcio-Ca e do Magnésio/Mg}

Foram colocados $10 \mathrm{~cm}^{3}$ de TFSA em erlenmeyer (125ml), adicionaram-se $100 \mathrm{ml}$ de extrator $\mathrm{KCl} 1,0$ $\mathrm{mol} / \mathrm{L}$. Agitou-se por $5 \mathrm{~min}$ em agitador circular horizontal a $200 \mathrm{rpm}$, deixando em repouso por, aproximadamente, $16 \mathrm{~h}$. A dosagem foi feita retirando uma alíquota de $0,5 \mathrm{ml}$ e colocando-a em um tubo de ensaio, onde foram adicionados $10 \mathrm{ml}$ da solução de $\mathrm{SrCl} 2$ contendo $1.680 \mathrm{mg} / \mathrm{L}$ de $\mathrm{Sr}$. As amostras foram agitadas em tubo de ensaio para a realização da leitura em espectrofotômetro de absorção atômica.

\section{RESULTADOS E DISCUSSÕES}

A ocupação das áreas úmidas das chapadas no Triângulo Mineiro é uma exigência econômica, uma vez que as atividades agrossilvipastoris implantadas seguem o modo de produção atual, apresentado por Harvey (2011), em que a natureza é convertida em mercadoria e baseada no acúmulo em grande escala (OLIVEIRA; ROSOLEN, 2014). O raciocínio economicista empregado pela sociedade para gerir suas 
riquezas não consegue apreender toda a complexidade dos fenômenos naturais e o planejamento em curto prazo não permite o entendimento da dinâmica integrada dos elementos naturais dos ecossistemas.

A paisagem do Cerrado modificou-se nas últimas cinco décadas de forma profunda, em virtude, principalmente, do agronegócio, no qual a agricultura e a pecuária voltada para exportação geram impactos consideráveis no solo e na água principalmente.

É neste sentido que a expansão agrícola tem acarretado algumas alterações negativas ao longo do tempo nos ecossitemas, envolvendo problemas relacionados, entre outros, com a adição de produtos químicos no solo (FOLEY, 2005; TILMAN, 2001; MEA, 2005). Onde a análise da morfologia dos solos permitiu a caracterização dos horizontes naturais e antropizados no campo. Apresentando cores, estrutura e consistência distintas. Em todas as amostras, foi comum encontrar a presença de nódulos ferruginosos vermelhos e resistentes observados na Figura 4. Possivelmente foram formados ao longo dos ciclos paleoclimáticos que afetaram as paisagens do Cerrado (OLIVEIRA et al., 2013). As cores apresentadas sugerem condições de hidratação e de saturação prolongadas e a estrutura maciça se forma em razão do alto teor de argila. Há um aumento da consistência quando o solo é drenado e usado para o cultivo.

As áreas úmidas das chapadas são caracterizadas, geralmente, pela quantidade elevada de argila encontrada na superfície e na subsuperfície, o que nfluencia outras propriedades físico-químicas desses solos (BISPO et al., 2011; SOARES, 2012).

A análise comparativa global da concentração dos elementos e dos parâmetros analisados (teste de Mann-Whitney) indicou que, no Murundu Drenado (MD), houve alterações significativas $(P<0,05)$ quando comparado com o Murundu de Referência (MR) em relação ao Cálcio $\left(\mathrm{Ca}^{++}\right)$e ao Magnésio $\left(\mathrm{Mg}^{++}\right)$.

No Murundu de Referência (MR), as correlações obtidas entre $0-20 \mathrm{~cm}$ foram diferentes daquelas entre $20-40 \mathrm{~cm}$ e $40-60 \mathrm{~cm}$. Nas duas últimas profundidades, as mesmas correlações foram encontradas. Essa distribuição sugere que existem propriedades e características dos solos que são determinantes na superfície e que elas distinguem essa camada de solo das demais. Uma vez que os solos dos murundus estudados são naturalmente pobres em decorrência de sua origem pedogenética, no que se refere aos cátions básicos e essa propriedade se reflete no complexo adsorvente. São solos formados a partir de intenso intemperismo, processo que envolve a lixiviação de cátions básicos e sílica e enriquece o solo em caulinita, oxihidróxidos de ferro e alumínio associados com quartzo residual.

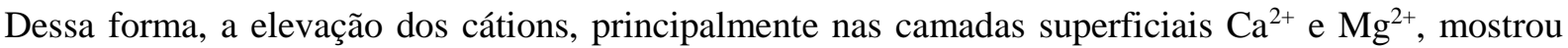
estar relacionada com manejo agrícola, que contribui para a elevação da concentração química e das mudanças dos parâmetros físicos-químicos dos solos analisados.

As mudanças ocasionadas em relação à concentração dos elementos citados foram principalmente devidas à adição de agrotóxicos, de fertilizantes e de corretivos do solo, especificamente na área drenada. 


\section{OS DESAFIOS DA GEOGRAFIA FÍSICA NA FRONTEIRA DO CONHECIMENTO \\ Instituto de Geociências - Unicamp \\ Campinas - SP \\ 28 de Junho à 02 de Julho de 2017}

A presença de teores mais elevados de Ca (cálcio) e de $\mathrm{Mg}$ (magnésio) no murundu drenado pode estar relacionada com a calagem, realizada para neutralizar a acidez do solo. Essa prática é comum e necessária nos solos do Cerrado, em razão da elevada acidez representada pelo hidrogênio e pelo alumínio. A neutralização com calcário dolomítico tem como objetivo substitui o $\mathrm{H}^{+}$e o $\mathrm{Al}^{3+}$ pelos cátions cálcio $(\mathrm{Ca})$ e de magnésio $(\mathrm{Mg})$, mecanismo que ajuda a elevar a CTC dos solos (VAN RAIJ, 2011).

No Murundu Drenado (MD), entre 0-20cm de profundidade, há forte e positiva correlação entre Ca e $\mathrm{Mg}$. Entre $20-40 \mathrm{~cm}$ também houve uma correlação positiva. Entre $40-60 \mathrm{~cm}$ as correlações foram mais negativas. Na profundidade 0-20, verificou-se que elementos como o $\mathrm{Ca}$ e o $\mathrm{Mg}$ possuem uma alta correlação, o que pode ser devido ao fato de que as amostras analisadas em questão são de um solo ácido que necessita de adição de corretivos de acidez do solo. Tal ação é realizada com elementos básicos como os citados que possuem capacidade de neutralização.

A troca de bases, têm uma relação muito próxima reativa nessa profundidade em virtude das reações entre os elementos ácidos e básicos.

Tabela 1 - Concentrações de Calcio e Magnésio em Murundu Referência (MR)

\begin{tabular}{c|c|c}
\hline Elementos & Cálcio $\left(\mathrm{Ca}^{2+}\right)$ & Magnésio $\left(\mathrm{Mg}^{2+}\right)$ \\
\hline Profundidades $(\mathrm{cm})$ & 0,07 & 0,04 \\
P1/0-20 & 0,05 & 0,03 \\
P1/20-40 & 0,03 & 0,02 \\
P1/40-60 & 0,09 & 0,05 \\
P2/0-20 & 0,04 & 0,04 \\
P2/20-40 & 0,07 & 0,02 \\
P2/40-60 & 0,10 & 0,04 \\
P3/0-20 & 0,05 & 0,04 \\
P3/20-40 & 0,03 & 0,01 \\
P3/40-60 & & \\
\hline
\end{tabular}

Tabela 2 - Concentrações de Cálcio e Magnésio em Murundu Drenado (MD)

\begin{tabular}{c|c|c}
\hline Elementos & Cálcio & Magnésio \\
\hline Profundidades $(\mathrm{cm})$ & 3,98 & 1,03 \\
P4/0-20 & 0,64 & 0,15 \\
P4/20-40 & 0,32 & 0,06 \\
P4/40-60 & 5,53 & 1,43 \\
P5/0-20 & 0,88 & 0,19 \\
P5/20-40 & 0,32 & 0,06 \\
P5/40-60 & 2,86 & 0,59 \\
P6/0-20 & 0,61 & 0,10 \\
P6/20-40 & 0,46 & 0,07 \\
P6/40-60 & 3,10 & 0,57 \\
P7/0-20 & 0,47 & 0,10 \\
P7/20-40 & 0,41 & 0,07 \\
P7/40-60 & & \\
\hline
\end{tabular}




\section{CONSIDERAÇÕES}

A expansão do agronegócio impulsionou a invasão de áreas úmidas que deveriam ser consideradas como áreas de preservação permanente, no entanto, a falta de uma legislação rigorosa e específica permitiu que grande parte dessas áreas fosse degradada. A retirada da vegetação original de Cerrado foi substituída, inicialmente, pela silvicultura e, mais recentemente, pelas commodities (soja, milho e feijão) e, como consequência, o uso intenso de produtos agroquímicos (agrotóxicos, fertilizantes e corretivos do solo). Tal situação tem ocorrido não só no bioma do Cerrado, mas, praticamente, em todo o território nacional visando ao aumento da produção e, consequentemente, ao aumento dos lucros de grandes empresas.

Os resultados analíticos permitiram constatar que houve alterações nas concentrações de alguns elementos, quando comparados os solos dos dois murundus (MD) e (MR). Houveram alterações significativas em relação ao cálcio $(\mathrm{Ca})$ e ao magnésio $(\mathrm{Mg})$. $\mathrm{O}$ uso dos agroquímicos por um longo período de tempo e em grande quantidade certamente contribuiu para que tais alterações fossem comprovadas. A necessidade de correção (calagem) do solo aferiu nas concentrações das bases.

Além da comparação realizada entre os dois murundus (MD) e (MR), foram comparadas as concentrações e possíveis alterações entre as profundidades deles. Quando comparadas as diferentes profundidades $(0-20,20-40$ e 40-60cm), estas bases tiveram variação significativa na estatística.

Tal variação pode ser justificada, no caso do Ca e Mg pela alteração pela adição de produtos corretivos da acidez do solo, haja vista que o solo dessa área é caracterizado como sendo ácido com grande presença de $\mathrm{H}^{+}$e $\mathrm{Al}^{+}$.

\section{REFERÊNCIAS}

BENNETT, E. M., CARPENTER, S. R.; CARACO, N. F. Human impact on erodable phosphorus and eutrophication: a global perspective. Bioscience 51, 227-234 (2001).

BORGES, F. A. Caracterização temporal das áreas úmidas e de preservação permanente da porção de alto e médio curso da bacia hidrográfica do Rio Uberabinha - MG com a aplicação de técnicas de geoprocessamento. 135f. Dissertação (Mestrado em Geografia) - Instituto de Geografia, Universidade Federal de Uberlândia, Uberlândia, 2012.

BRITO, J. L. S. Adequação das potencialidades do uso da terra na Bacia do ribeirão Bom Jardim no Triângulo Mineiro (MG): Ensaio de Geoprocessamento. São Paulo: USP/FFLCH, 2001, 184 p

CANFIELD, D. E., GLAZER, A. N.; FALKOWSKI, P. G. The evolution and future of earth's nitrogen cycle. Science 330, 192-196 (2010). 
COSTA, F. P. M. Utilização de sistemas de informação geográfica na identificação de áreas vulneráveis à contaminação do lençol freático: o caso da bacia do alto e médio curso do Rio Uberabinha - MG. 2009. 136 f. Dissertação (Mestrado em Geografia) - Instituto de Geografia, Universidade Federal de Uberlândia, Uberlândia, 2009.

DIAZ, R. J.; ROSENBERG, R. Spreading dead zones and consequences for marine ecosystems. Science 321, 926-929 (2008).

FERREIRA, D. A. Concentração de macro e micronutrientes em áreas úmidas de chapada no município de Uberlândia/MG. 2015- 126f. Dissertação (Mestrado em Geografia) - Instituto de Geografia, Universidade Federal de Uberlândia, Uberlândia, 2009.

FOLEY, J. A. Global consequences of land use. Science v. 309, p. 570-574 2005.

FRIEDLINGSTEIN, P., BOPP, L.; P. J., CIAIS; L. DUFRESNE; H. FAIRHEAD; P. LETREUT; MONFRAY; ORR, J. (2010), Positive feedback between future climate change and the carbon cycle, Geophys. Res. Lett., 28(8), 1543-1546

GIBBS, H.K., RUESCH, A.S., ACHARD, M.K., CLAYTON, M.K., HOLMGREN, P., RAMANKUTTY, N., FOLEY, A. (2010). Tropical forests were the primary sources of new agricultural land in the 1980s and 1990s. Proceedings of National Academy of Sciences 107, no.38, 16732-16737 (2010).

GLEICK, P. H. Global freshwater resources: soft-path solutions for the 21st century. Science $302,1524-1528$ (2003).

GUPTA, S.K.; Assessment of ecotoxicological risk of accumulated metals in soils with the help of chemical methods standardized through biological tests. In: VERNET, J.P. Heavy metals in the environment. Amsterdam: Elsevier, 1991. 55-65.

MACHADO, H. A. Comportamento do carbono orgânico em área úmida do Cerrado: Estudo de caso em uma área úmida do Córrego Beija-Flor. Dissertação (Mestrado em Geografia) - Instituto de Geografia, Universidade Federal de Uberlândia, Uberlândia, 2012.

MATIZA T. Wetlands in Zimbabwe: an overview. In: MATIZA T, CRAFTER SA (eds). Wetlands Ecology and Priorities for Conservation in Zimbabwe: Proceedings of a Seminar on Wetlands Ecology and Priorities for Conservation in Zimbabwe, Hare Kentucky Airport Hotel, 1994, 170p.

MATSON, P., PARTON, W., POWER, A. \& SWIFT, M. Agricultural intensification and ecosystem properties. Science 277, 504-509 (1997).

MITSCH, W., GOSSELINK, J.,Wetlands . The IUCN Wetlands Programme. Published by IUCN, Switzerland, pp 3-10 (2007), Wiley, New York. 
NIERING, W. A. Wetlands. New York: The Audubon Society, 1985. 638 p

OLIVEIRA, D. A. As áreas de preservação permanente em topo de chapada e a adequação à legislação ambiental. f135. Dissertação (Mestrado em Geografia) - Instituto de Geografia, Universidade Federal de Uberlândia, Uberlândia, 2013.

POSTEL, S. Pillar of Sand; Can the Irrigation Miracle Last? W.W. Norton \& Company, New York, 1999.

QUEIROZ, A. T. Análise e avaliação da demanda e da disponibilidade hídrica nos alto e médio curso do Rio Uberabinha e o abastecimento público em Uberlândia (MG). 2012. 138 f. Dissertação (Mestrado) -Curso de Geografia, Departamento de Instituto de Geografia, Universidade Federal de Uberlândia, Uberlândia, 2012. Disponível em: <http://www. ppgeo. ig. ufu. br/sites/ppgeo. ig. ufu. br/files/Anexos/Boo page/Arlei\%20Teodoro\%20de\%20Queiroz. pdf>. Acessado em: 10 jan. 2014

RAMOS, M. V. V. ; CURI,N. ; MOTTA,P. E. F.; VITORINO, A. C. T.; Ferreira, M. M.; SILVA, M. L. N. Veredas do Triângulo Mineiro: Solos, água e uso. Ciência Agrotécnica, Lavras, v. 3,n. 2, p. 283-293, 2006.

RESENDE, T. M. Conversão de uso e potencial de estoque do carbono nos diferentes usos do solo e cobertura vegetal na bacia do ribeirão Bom Jardim no Triângulo Mineiro (MG). 142f. Dissertação (Mestrado) - Programa de Pós Graduação em Geografia, Departamento de Instituto de Geografia, Universidade Federal de Uberlândia, Uberlândia, 2011.

ROSEGRANT, M. W., S. MEIJER,; S. A. CLINE. 2002. International model for policy analysis of agricultural commodities and trade (IMPACT): Model description. Washington, D.C.: International Food Policy Research Institute.

ROSOLEN, V. - Os Sistemas Úmidos na Paisagem de Chapada, o Uso da Terra e Desafios da Preservação Ambiental., v. 15, n² 2, p. 221-230, 2014.

SCHNEIDER, M. O. Bacia do Rio Uberabinha: Uso agrícola do solo e meio ambiente. 1996. 157f. Tese (Doutorado) - Departamento de Geografia da Faculdade de Filosofia, Letras e Ciências Humanas, Universidade de São Paulo, São Paulo, 1996

SOARES, Â. M. A dinâmica hidrológica na bacia do alto curso do Rio Uberabinha - Minas Gerais. 2008. 227f. Tese (Doutorado) - Pós-Graudação em Geografia, Universidade Federal de Uberlândia, Uberlândia, 2008.

TILMAN, D. Forecasting agriculturally driven global environmental change. Science. v. 292, p. 281-284. 2001. 
VITOUSEK, P. M., MOONEY, H. A., LUBCHENCO, J.; MELILLO, J. M. Human domination of Earth's ecosystems. Science 277, 494-499 (1997).

VOROSMARTY, C. J., GREEN, P., SALISBURY, J. \& LAMMERS, R. B. Global water resources: vulnerability from climate change and population growth. Science 289, 284-288 (2000) 